\title{
Emotional model for a multi-robot system with emergent behavior
}

\author{
Angel Gil ${ }^{1}$, Jose Aguilar ${ }^{2}$, Eladio Dapena ${ }^{3}$, Rafael Rivas ${ }^{4}$ \\ ${ }^{1}$ Laboratorio de Prototipos, Decanato de Investigación, Universidad Nacional Experimental del Táchira, Venezuela \\ ${ }^{1,2}$ Tepuy R+D Group, Artificial Intelligence Software Development, Venezuela \\ ${ }^{2}$ CEMISID, Universidad de Los Andes, Venezuela \\ ${ }^{2}$ CIDITIC, Universidad EAFIT, Medellín, Colombia \\ ${ }^{3,4}$ LASDAI, Facultad de Ingeniería, Universidad de Los Andes, Venezuela
}

\begin{tabular}{l} 
Article Info \\
\hline Article history: \\
Received Oct 28, 201 \\
Revised May 23, 2020 \\
Accepted Jun 8, 2020 \\
\hline Keywords: \\
Emergent behaviors \\
Emotions \\
Multi-robot systems \\
Robotics \\
Self-organization
\end{tabular}

Article Info

Received Oct 28, 2019

Revised May 23, 2020

Accepted Jun 8, 2020

\section{Keywords:}

ergent behaviors

Multi-robot systems

Self-organization

\begin{abstract}
This article describes an emotional model for a general-purpose robot operating in a multi-robot system with emergent behavior. The model considers four basic emotions: anger, rejection, sadness and joy, plus a neutral emotional state, which affect the behavior of the robot, both individually and collectively. The emotional state of each robot in the system is constructed through the conjunction of a series of factors related to their individual and collective actions, which are: safety, load, acting and interaction, which serve as input to an emotional process that results in an index of satisfaction of the robot that establishes the emotional state in which it is in a certain moment. The emotional state of a robot influences its interactions with the other robots and with the environment, that is, it determines its emergent behavior in the system. This paper presents the design of this model, and establishes some considerations for its implementation.
\end{abstract}

This is an open access article under the $\underline{C C B Y-S A}$ license.

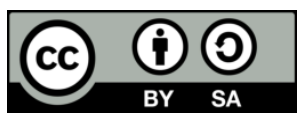

Corresponding Author:

Jose Aguilar, TEPUY R+D Group,

Mérida, Venezuela.

Email: aguilar@ula.ve

\section{INTRODUCTION}

Emotions are psycho and physiological reactions that occur in individuals and that condition their action in certain situations. Emotions condition the body how it perceives a stimulus of the environment and how it acts in function of it. The emotional spectrum in humans is very broad, and affects positively or negatively their performance in the environment.

Multiple works have been developed in the area of emotions for robots that include model proposals, hardware implementations, and human-robot interactions based on emotions, among others. In [1], emotions are included in a multi-robot system with the object of generating intelligent behaviors between robots that cooperate in the execution of a specific task. In [2] is presented a hardware design for the implementation of emotions in a robotic agent. In [3], the authors propose an emotional model that considers multiple factors that affect emotions: stimuli, cognitive and non-cognitive factors, personality, the subconscious of the agent, among others. [4] describes an emotional model for a pet robot, in order to adapt it to have a natural behavior. In [5] is presented the study of emotions for robots in two domains: the first related to the way in which robots relate to humans, and the second related to the robot's adaptation to the environment and how emotions can improve its performance. In general, the emotions in the robots are represented in discrete states that control the actions of these during their operation. 
This research proposes an emotional model that considers four basic emotions, which influence the behavior of a general-purpose robot, through the direct involvement in its processes of perception and performance, as well as in its decision making. The model is implemented in an architecture for multi-robot systems with emergent behavior. The emotions influence the interactions that occur between robots and between robots and the environment. Even though they are not able to recognize the emotions of others, their responses to interactions with other robots in the system or with the environment vary according to their emotional state. In this way, the current emotional state in a robot defines its emergent behavior in the system.

The main difference with previous works is that we propose a general emotional model, which is used in order to generate emergent behaviors in the robots of a multi robot system. In our case, the emotions of the robots describe their current functional states, and they are used by the multi robot system in order to define the actions of the robots.

This article is organized in 4 sections. The first one describes the proposed emotional model. The second refers to its implementation. The next section presents the results of the tests carried out. To finalize, the conclusions of the research are presented.

\section{EMOTIONAL MODEL FOR AN ARCHITECTURE FOR MULTI-ROBOT SYSTEMS WITH EMERGENT BEHAVIOR}

In [6-9] is presented an architecture for multi-robot systems with emergent behavior (called AMEB), which is structured in three levels: one individual, one collective and another for the knowledge and learning management (see Figure 1). At the individual level of AMEB, a behavioral module is implemented in [10], which aims to manage the behavior of the robot. This behavioral module requires an emotional model, which considers the set of next emotions $\varepsilon=$ \{anger, rejection, neutral, sadness, joy $\}$, represented in a one dimensional space proposed in [11-13], where the $X$ axis represents the satisfaction or dissatisfaction state of the robot in the interval [-1,1] (see Figure 2), and the emotional spectrum ranges from highly negative emotions like anger, to highly positive emotions such as joy, plus a neutral or non-emotion. Three sub-intervals are defined based on the relationship between emotions and individual behaviors [11-13]: an interval related to reactive behaviors, one related to cognitive behaviors, and a third interval related to collective behaviors.

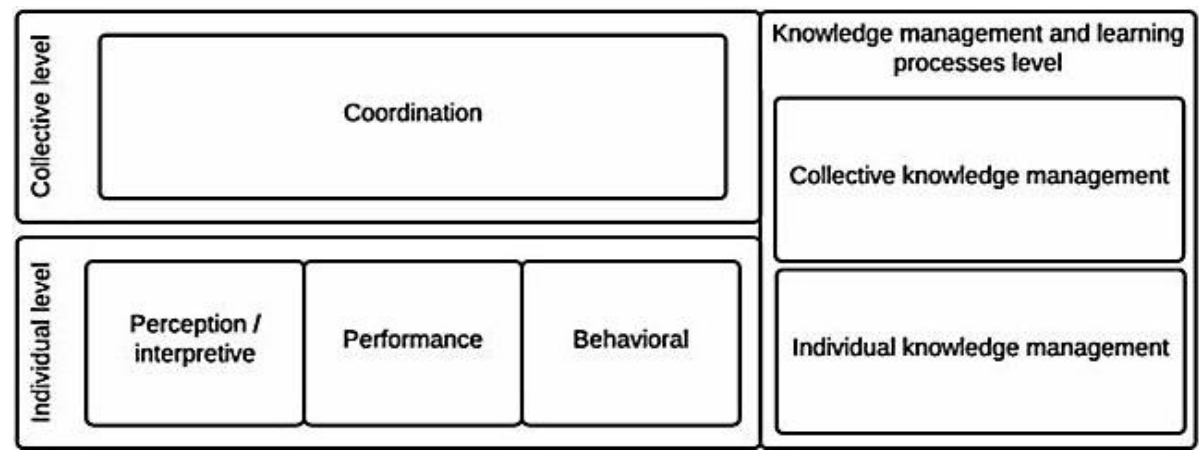

Figure 1. AMEB Architecture [6, 8, 9]

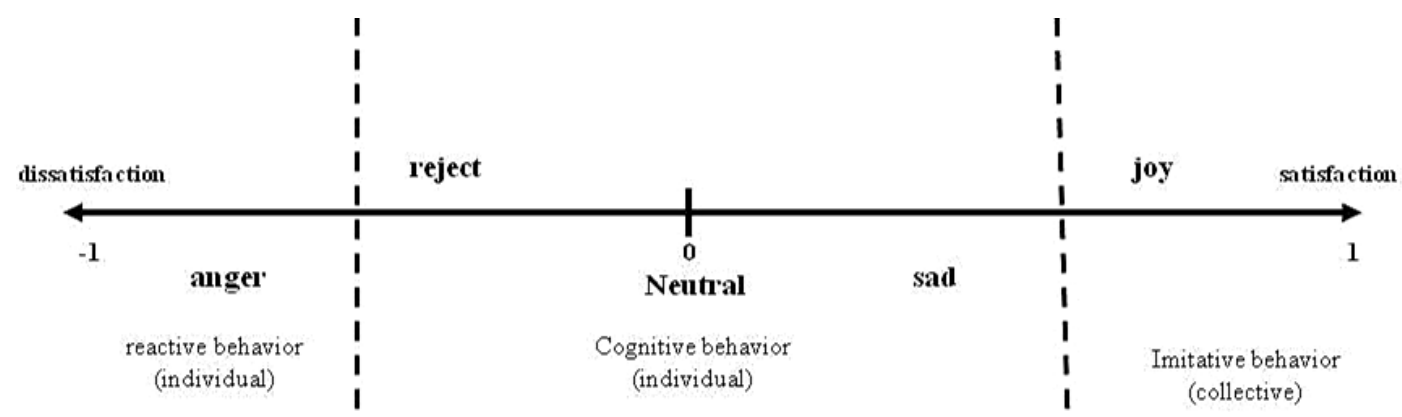

Figure 2. Emotional model 
The level of satisfaction of the robot is influenced by a series of factors that define the internal state of the robot, and that are related to the operation of the robot and its performance in the environment [10]:

- Battery state $(B S)$ : is represented by the energy level of the robot's battery at an instant $t$.

$$
B S_{t}=\frac{n c_{t}}{c b}
$$

where: $n c_{t}=$ level of charge in an instant $t, c b=$ Battery charge capacity.

- Operating state $(O S)$ : represents the level of performance of the robot. It is given by the actual time of action $(t a)$ of the robot versus the total system performance time $(t t)$.

$$
O s_{\mathrm{t}}=\frac{\mathrm{ta}}{\mathrm{tt}}
$$

- Security state (SS): defined by the number of collisions (c) (caused by other robots or obstacles) and failures $(f)$ versus the total system performance time $(t t)$.

$$
\mathrm{SS}_{\mathrm{t}}=\frac{\mathrm{c}+\mathrm{f}}{\mathrm{tt}}
$$

- Interaction state (IS): it is defined by the social or interaction capacity of the robot. It is measured by the number of messages sent $(m e)$ or received $(m r)$ versus the total system performance time $(t t)$.

$$
\mathrm{IS}_{\mathrm{t}}=\frac{\mathrm{m}_{\mathrm{e}}+\mathrm{m}_{\mathrm{r}}}{\mathrm{tt}}
$$

The robot perceives stimuli that influence the state of the mentioned parameters. This activates the emotional process of the robot, which generates a satisfaction index $(S I)$ that defines the current emotion in the robot and the type of behavior associated (see Figure 3). In [11-15] are considered three types of behavior: imitative, cognitive and reactive, which are related to a specific emotional state. In our proposal, the same assumptions are made: negative emotions predispose the individual to problem solving through a process that goes from the local to the collective, while the positive emotions lead to global approaches, ranging from the collective to the individual. Table 1 presents the emotions considered in this model, their classification, and the type of associated behavior.

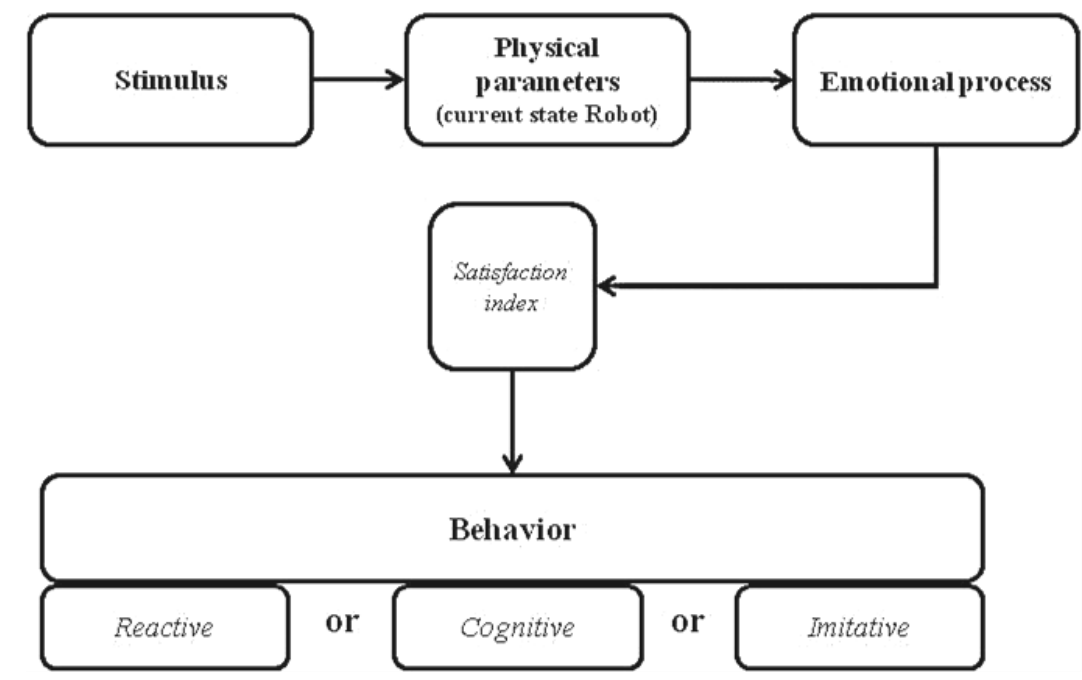

Figure 3. Emotional process 
Table 1. Relationships between the Emotions and the Behaviors

\begin{tabular}{ccl}
\hline Emotion & Classification & Behavior \\
\hline Joy & Highly positive & Imitation \\
Sadness & Slightly positive & Cognitive \\
Neutral & - & - \\
Reject & Slightly negative & Cognitive \\
Anger & Highly negative & Reactive \\
\hline
\end{tabular}

As seen in the Table 1, highly positive emotions, such as joy, predispose the individual to imitate the behavior of other team members at the moment of deciding, while the slightly positive, such as sadness, lead to a process where the cognitive aspect predominates over the imitative aspect. Also, slightly negative emotions lead the individual to a process of internal reflection for decision making, and finally, a highly negative emotion as anger, leads to behaviors that are clearly reactive, where the individual seeks to achieve their survival by reacting to the stimuli of the environment without previous reasoning. The emotions affect the parameters of the robot; for example, if the robot is sad, then its ability of feeling will be affected; or if the robot is happy, then its speed of movement will be greater, which will affect its perform at a given time.

The types of behavior are defined below.

a. Imitative: behaviors where an individual seeks to behave in a similar way to another, for example: persecution.

b. Cognitive: behaviors where there is a reasoning process in order to decision-make, by the individual, example: shelter search.

c. Reactive: behaviors where the individual seeks to survive, for example: obstacle avoidance.

The inclusion of emotions in the multi-robot system seeks to improve its adaptability to the dynamics of the environment, as well as facilitate the emergence in the system, by modifying the way of performing the behaviors that each individual is able to execute. The conjunction of individual behaviors defines the overall behavior of the system, which by its nature cannot be predicted a priori [16].

\section{IMPLEMENTATION OF THE MODEL}

The model is implemented in the AMEB behavioral module described in [10], which is structured in four layers (see Figure 4), in which the processes involved in the emotional model are organized: a) reactive layer: manages the reactive behaviors of the robot, which are generated by a stimulus - reaction process; b) cognitive layer: deliberative behaviors are managed in the robot, based on their local knowledge; c) social layer: it exploits the collective knowledge in the processes of decision making of the robot, basically, it manages the way that the robot interacts with other robots in the system, as well as its associated behaviors; d) affective layer: is responsible for managing the emotional process of the robot. The phases involved in the emotional model are described below, based on the phases described in [11].

a. Classifier

In the proposed model, we define four basic emotions and a neutral or non-emotional state, considering positive and negative emotions, which lead to individual or collective behaviors. In this phase the types of emotion that the robot can activate at a certain time are defined.

b. Behavior model

The reactive, cognitive and social layers implement a set of behaviors of the robot in the environment [10]. These behaviors are constructed by the conjunction of two or more basic robot skills, which are closely related to their hardware and software capabilities, some of these skills are:

- Move: The robot moves forward or backward, it can be for a certain time or a certain distance.

- Rotate: The robot rotates in one direction for a given time or a given number of degrees.

- Perceiving: The robot perceives information of the environment through its sensors, as well as information about its internal state.

- Stop: The robot can stop at any moment.

From these skills are built behaviors of greater complexity, which define the way the robot acts, among which are:

- Explore: Through this behavior the robot is able to explore the environment at random.

- Obstacle avoidance: allows the robot to evade obstacles, which can be fixed or moving objects.

- Pursuit: it allows the robot to pursue for some time another robot.

- Shelter: the robot seeks a safe area (represented by marks on the ground), and stay in it until reaching the desired state.

- Confrontation: Depending on your emotional state, the robot may face another, when a conflict arises for a resource. 
- Recharge: The robot has the ability to satisfy its energy level through a search mechanism of a charge zone.

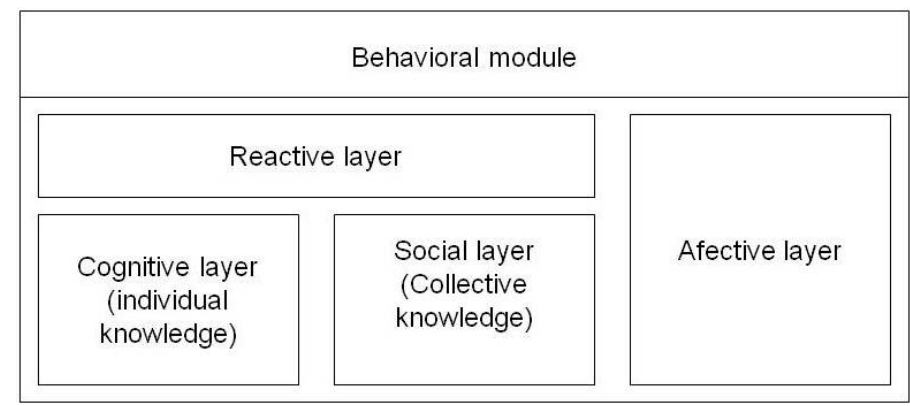

Figure 4. AMEB Behavioral Module

These behaviors are associated, as mentioned above, to a specific emotional state, for which the following rules are defined [10]:

Rule 1: If <emotional state> is highly positive. then <imitative_behavior>

Rule 2: If <emotional state> is positive, then <imitative_behavior>

Rule 3: If <emotional state> is slightly positive, then <cognitive_behavior_priority>

Rule 4: If <emotional state > is neutral, then <behavioral>

Rule 5: If <emotional state> is slightly negative, then <cognitive_behavior_priority>

Rule 6: If <emotional state> is negative, then <cognitive-behavior_priority>

Rule 7: If <emotional state> is highly negative, then <reactive_priority>

a. Emotional configurator

Determining the emotion of the robot at an instant $t$ is a fundamental stage of the emotional process. It depends on the level of satisfaction of the robot, which is given by the factors described in the previous section. Below, it is described this process.

- Evaluation of the occurrence of an external or internal stimulus that affects the parameters involved in the robot satisfaction index $(S I)$.

- Determination of the values of the parameters.

- Calculation of the SI satisfaction index using a fuzzy model.

- Determination of the current emotion.

In this phase, the satisfaction index is calculated using a fuzzy system composed of four input variables and one output variable. The input variables, as has been explained in the previous section, represent the internal state of the robot, and are related to operating parameters. The output variable is related to the satisfaction status of the robot, and is associated with an emotion:

- Input variables: $B S, O S, S S, I S$.

- Output variable: $S I$.

The input variables have three fuzzy sets: \{low, normal, high $\}$ and the output variable has five fuzzy sets: $\{$ anger, reject, neutral, sadness, joy\}. Some of the rules that govern the system are:

Rule 1: If BS is low and OS is low and SS is high and IS is low, then SI is sadness.

Rule 2: If BS is low and OS is low and SS is normal and IS is low, then SI is sadness.

Rule 3: If BS is low and OS is normal and SS is normal and IS is low, then SI is sadness.

Rule 4: If BS is low and OS is normal and SS is normal and IS is normal, then SI is rejected.

Figure 5 shows an example of the implementation of the fuzzy model in MATLAB®. For the case of the Figure 5, for the set of input \{BS: high,OS: normal, SS: low, IS: high\} was obtained the next output: \{joy\}. This, according to our model describes the following:

- Battery Charging: optimal.

- Operation state: normal, the robot has been executing some task during a good part of the operating period of the global system.

- Safety factor: Low, indicating that there are few or no collisions and/or blockages.

- Interaction factor: high, the robot has interacted with the other team members.

The robot at that moment is satisfied and the active emotion is joy.

Figure 6 shows the relationship between the battery state (BS) and state of operation (OS) variables with the other system variables, and how they influence the output variable (SI). It can be observed, for

Int J Rob \& Autom, Vol. 9, No. 3, September 2020 : $220-232$ 
example, that for normal values of BS and OS without considering the other states, the satisfaction index can reach a maximum value close to normal, implying that the active emotion would be slightly positive.

b. Behavior selection

In this phase is modified the current behavior of the robot, as a consequence of the previous phase. This modification involves the association of an emotion to a behavior, as is shown in Table 1, according to the rules established in phase 2. The robot's performance is affected by the current emotion in two specific aspects.

- The selected behavior (imitative, cognitive or reactive) of the set of behaviors of the robot.

- The performance parameters that are modified by the satisfaction index of the robot, which influence the way in that the behavior is executed.

The performance parameters that can be modified are related to the characteristics of the robots to be managed by the architecture [14]:

- velocity of displacement

- range of detection of objects in front

- range of detection of marks on the ground

- decision-making capacity (time to decide).

What implies that for the same behavior, different results will be obtained. For example: if the robot is in a chase and its current emotion is joy, then it will move faster and its sensory abilities will work better that if the robot has as current emotion the sadness.

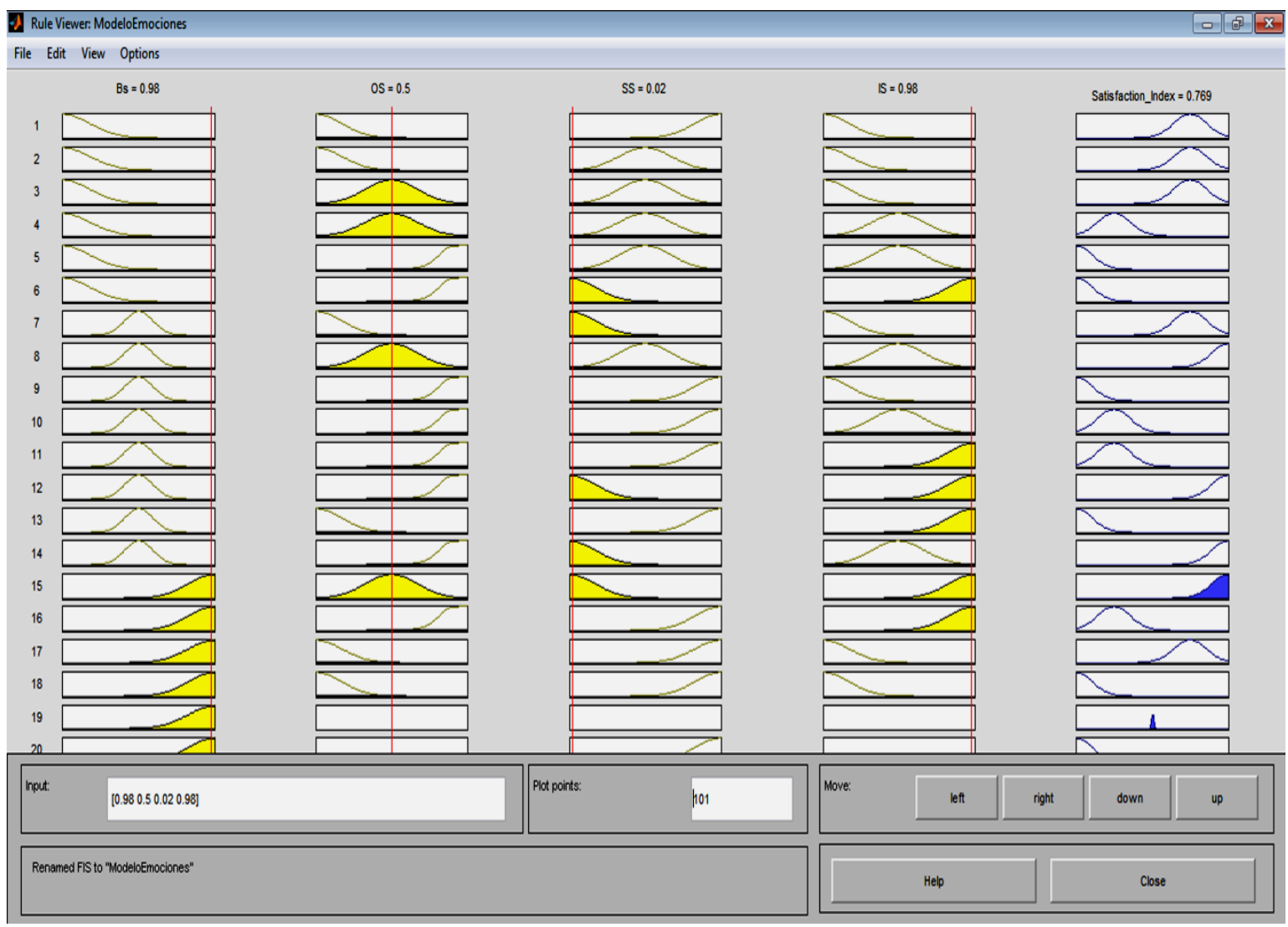

Figure 5. Implementation of the fuzzy model 

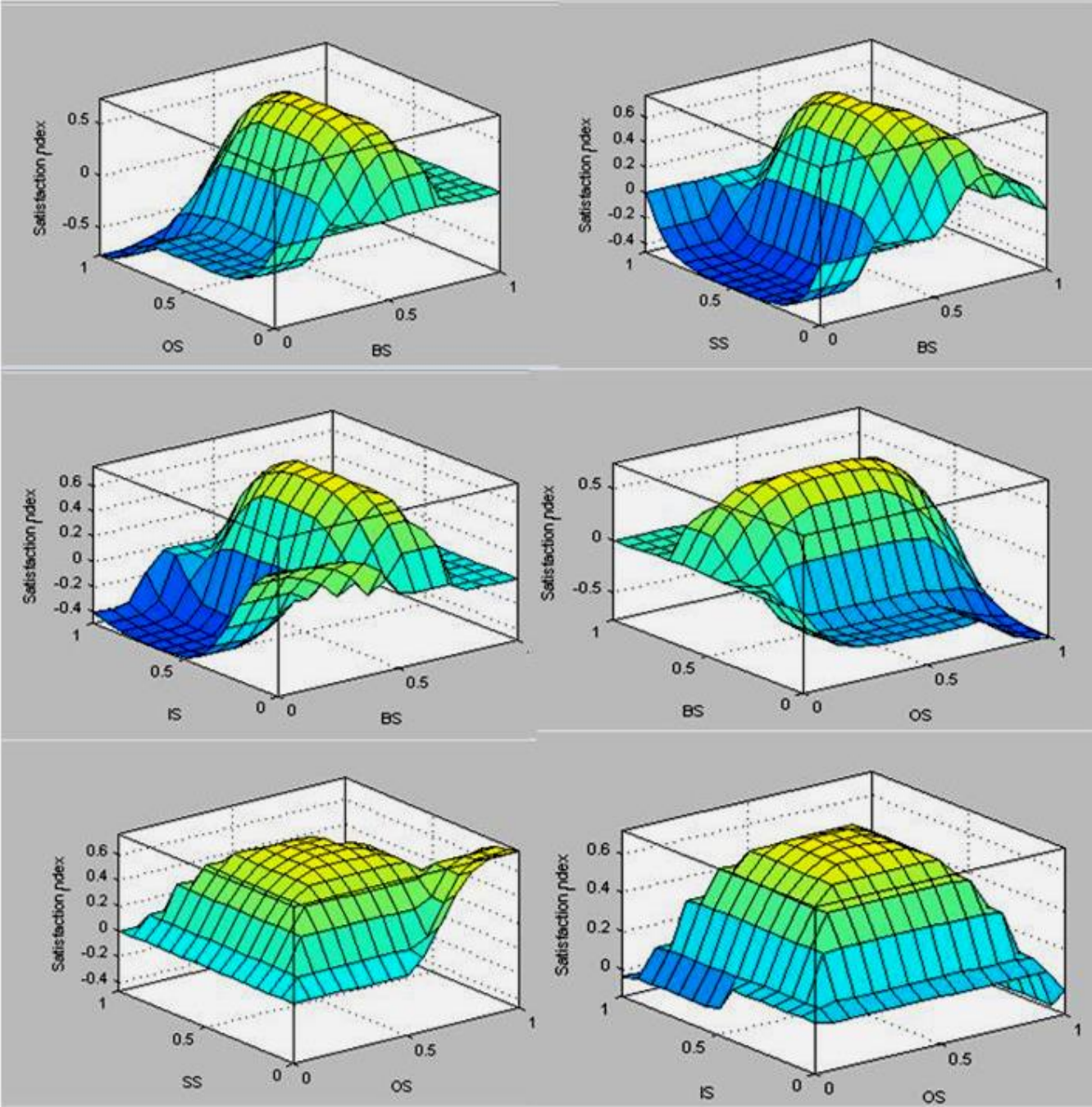

Figure 6. Relationship of the battery state and state of operation variables with the other factors and with the satisfaction index

\section{EXPERIMENTS}

\subsection{Scenarios}

Different tests were carried out, in order to verify the emotional model through the variation of the parameters in the implemented fuzzy system. In these tests, the aim is to verify the emotional model in the robots, not focusing on the overall behavior of the system. The interactions between the robots and of them with the environment are simulated, as well as the factors that alter their internal state. The experimental scenarios are the following:

- Determination of the current emotional state according to the current operational state of the robots

- Analysis of the emotional behavior of a robot according to the events in the environment.

a. First scenario

We present the result of the test for the first scenario, where randomly were generated values for the parameters that define the state of the robot, and verified that the active emotion was the expected. Table 2 summarizes the results obtained in the first scenario, in which the emotional state of a robot was verified, according to the factors that influence it. The BS, OS, SS and IS columns present the values of the factors that define the internal state of the robot. As mentioned above, they have three fuzzy sets \{low, medium, high . The SI column presents the value of the satisfaction index, which has five fuzzy sets \{anger, reject, neutral, sadness, joy\}, such that its result is related to an emotional state. For example, a robot model whose internal state is \{BS: high, OS: high, SS: low, IS: high \} has a high SI, so it is happy at that moment. 
Table 2. Determination of the current emotional state

\begin{tabular}{|c|c|c|c|c|c|c|}
\hline No. & BS & OS & SS & IS & SI & Emotion \\
\hline 1 & 0,7461 & 0,8801 & 0,108 & 0,7517 & 0,5575 & Joy \\
\hline 2 & 0,6625 & 0,4443 & 0,4599 & 0,3684 & 0,6125 & Joy \\
\hline 3 & 0,5233 & 0,7559 & 0,4509 & 0,9418 & 0,167 & Sadness \\
\hline 4 & 0,2599 & 0,6033 & 0,5511 & 0,0172 & 0,4222 & Sadness \\
\hline 5 & 0,962 & 0,7833 & 0,8054 & 0,8291 & $-0,5437$ & Anger \\
\hline 6 & 0,5402 & 0,1139 & 0,7009 & 0,6266 & $-0,0546$ & Reject \\
\hline 7 & 0,0303 & 0,9786 & 0,8722 & 0,5387 & $-0,5277$ & Reject \\
\hline 8 & 0,6963 & 0,8486 & 0,0522 & 0,6505 & 0,6423 & Joy \\
\hline 9 & 0,5197 & 0,0506 & 0,2197 & 0,7266 & 0,1388 & Sadness \\
\hline 10 & 0,059 & 0,4662 & 0,4596 & 0,0945 & 0,3895 & Sadness \\
\hline 11 & 0,89 & 0,3257 & 0,9585 & 0,8776 & $-0,7518$ & Anger \\
\hline 12 & 0,3302 & 0,6302 & 0,79 & 0,0144 & 0,321 & Sadness \\
\hline 13 & 0,2297 & 0,2303 & 0,4519 & 0,2943 & $-0,0485$ & Reject \\
\hline 14 & 0,1139 & 0,5799 & 0,3334 & 0,1799 & 0,2091 & Sadness \\
\hline 15 & 0,3109 & 0,6032 & 0,0591 & 0,9263 & $-0,0368$ & Reject \\
\hline 16 & 0,2284 & 0,5999 & 0,7409 & 0,0682 & 0,3733 & Sadness \\
\hline 17 & 0,652 & 0,4484 & 0,5068 & 0,5811 & 0,6194 & Joy \\
\hline 18 & 0,0662 & 0,0354 & 0,1999 & 0,6372 & $-0,405$ & Reject \\
\hline 19 & 0,2754 & 0,5138 & 0,4272 & 0,6513 & $-0,3906$ & Reject \\
\hline 20 & 0,2818 & 0,4077 & 0,1687 & 0,8646 & $-0,2095$ & Reject \\
\hline 21 & 0,056 & 0,775 & 0,279 & 0,2822 & $-0,1014$ & Reject \\
\hline 22 & 0,8169 & 0,9142 & 0,6754 & 0,976 & $-0,4362$ & Reject \\
\hline 23 & 0,5289 & 0,7826 & 0,9037 & 0,0364 & $-0,6097$ & Anger \\
\hline 24 & 0,6944 & 0,2955 & 0,9085 & 0,3262 & 0,004 & Reject \\
\hline 25 & 0,2124 & 0,1518 & 0,7472 & 0,973 & $-0,2692$ & Reject \\
\hline 26 & 0,5433 & 0,8479 & 0,2605 & 0,365 & 0,6661 & Joy \\
\hline 27 & 0,7025 & 0,7849 & 0,6896 & 0,3091 & $-0,2271$ & Reject \\
\hline 28 & 0,9564 & 0,2708 & 0,1318 & 0,1209 & 0,01 & Reject \\
\hline 29 & 0,4445 & 0,2278 & 0,1235 & 0,9158 & $-0,0441$ & Reject \\
\hline 30 & 0,0854 & 0,321 & 0,1909 & 0,1355 & 0,1272 & Sadness \\
\hline 31 & 0,2336 & 0,3972 & 0,7561 & 0,2524 & 0,2451 & Sadness \\
\hline 32 & 0,92 & 0,3257 & 0,9045 & 0,8776 & $-0,7518$ & Anger \\
\hline 33 & 0,7962 & 0,5707 & 0,0734 & 0,4996 & 0,2011 & Sadness \\
\hline 34 & 0,6912 & 0,5718 & 0,8223 & 0,6153 & $-0,1566$ & Reject \\
\hline 35 & 0,8491 & 0,9201 & 0,108 & 0,7517 & 0,5575 & Joy \\
\hline 36 & 0,9468 & 0,6991 & 0,9259 & 0,6983 & $-0,6792$ & Anger \\
\hline 37 & 0,5202 & 0,7963 & 0,4926 & 0,0293 & 0,0833 & Sadness \\
\hline 38 & 0,9538 & 0,4416 & 0,6549 & 0,5279 & $-0,4507$ & Reject \\
\hline 39 & 0,0736 & 0,4462 & 0,8901 & 0,0321 & 0,2451 & Sadness \\
\hline 40 & 0,207 & 0,4657 & 0,5385 & 0,8271 & $-0,4594$ & Reject \\
\hline
\end{tabular}

The fuzzy system establishes the expected emotions, according to the input variables about the states of the robot in a given a time $t$. The value of the index of satisfaction in each case shows how the level of intensity of the same emotion can vary. This could be used to affect the behavior of the individual at the time of performing a behavior, in other words, be more precise. Thus, the robots could present different levels of joy, and according to it, act different. Figure 7 shows different values for the same emotion in different robots in the first scenario. 


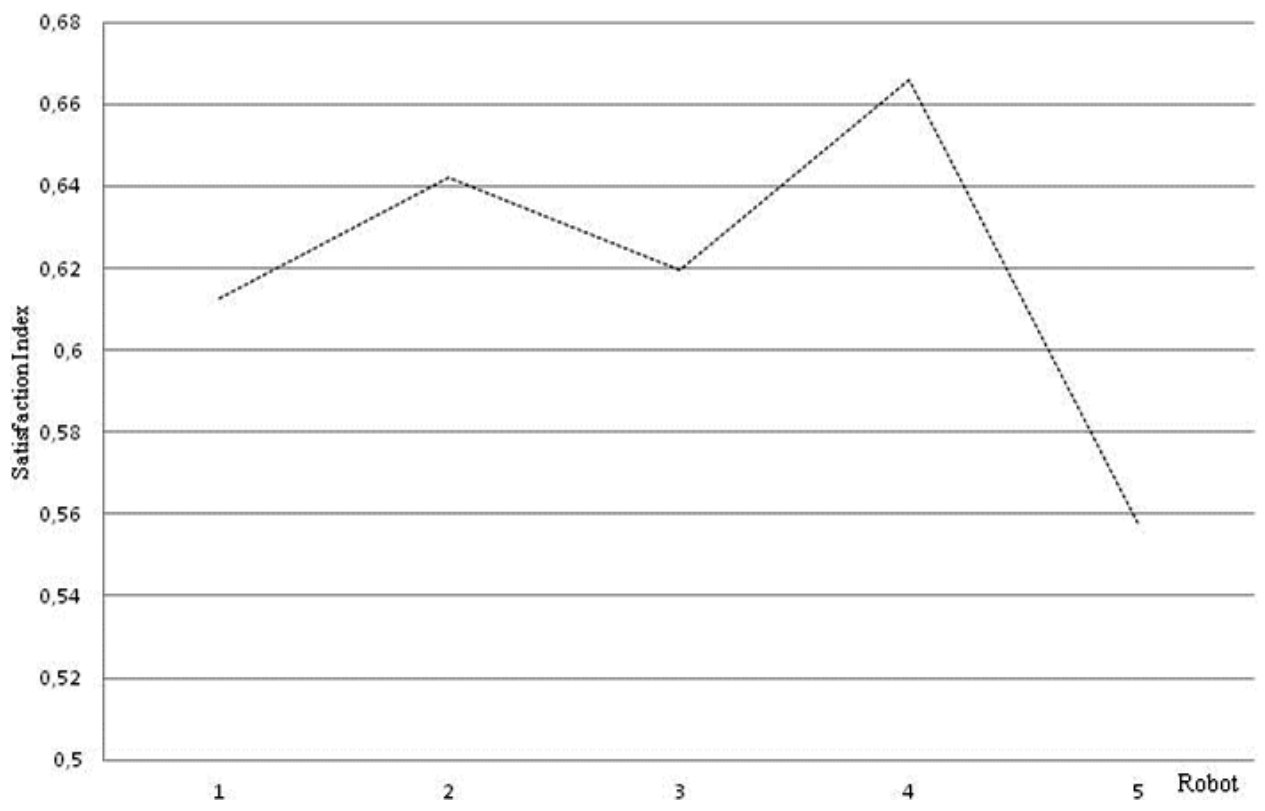

Figure 7. Levels of intensity of the joy emotion for 5 individuals

\section{b. Second scenario}

Below is presented the results of the second scenario, where the dynamics of a robot's emotional state during its operation are analyzed, for which the events that cause changes in its state are monitored: collisions/failures (C/F), the effective time of action (OT), interactions (I) and battery charge (BC). The robot interacts with the environment for a period of time $t$, and the samplings are carried out every 10 units of time. The simulation results are presented below, for which the first 100 samples carried out during time $t$ are taken into consideration.

Figure 8 shows the first results for the first group of samples, in a context where there is a set of negative factors at this time in the environment, such as collisions, isolation of the robots, etc. Figure 8 shows that, for $\mathrm{t}=0$, the robot is in a neutral state. During the beginning of its performance, the robot presents an abrupt tendency towards a highly negative emotion, this is motivated by the fact that the received stimuli were negative (high number of collisions, few interactions, etc.). During its operation, it begins to change its state to a highly positive emotional state, then the emotional state begins to decline, and it stabilizes in a slightly negative state. This is due to the occurrence of factors over time that negatively influence the state of the robot.

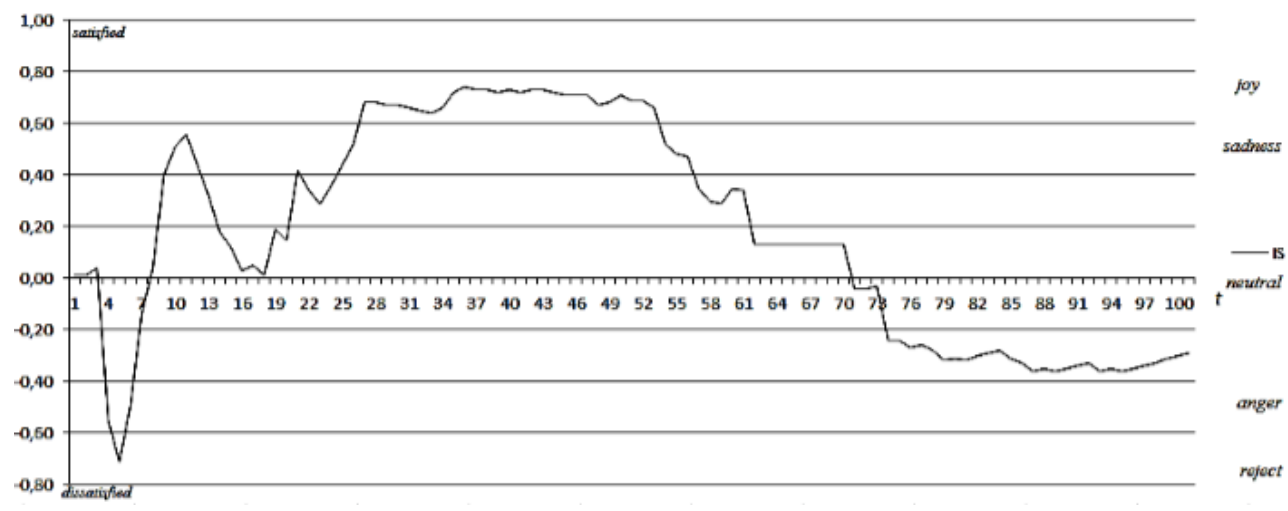

Figure 8. Results for the first test for the second scenario

Figures 9 and 10 show the results of the second test. In Figure 9, it is observed that at the beginning, the robot had few interactions (I), concentrated stops in its operation (OT) in a short interval of time, and 
the discharge rate was similar, in addition to a high number of collisions (C). This should cause that the emotional state should be highly negative emotion. With the passage of time, the social capacity of the robot should increase steadily, the collisions should increase slowly stabilizing, the discharge rate should low, and also the number of stops. These events should influence that the robot maintains a positive emotional state at the end (high interactions, safe operation and stable battery charge (BC)).

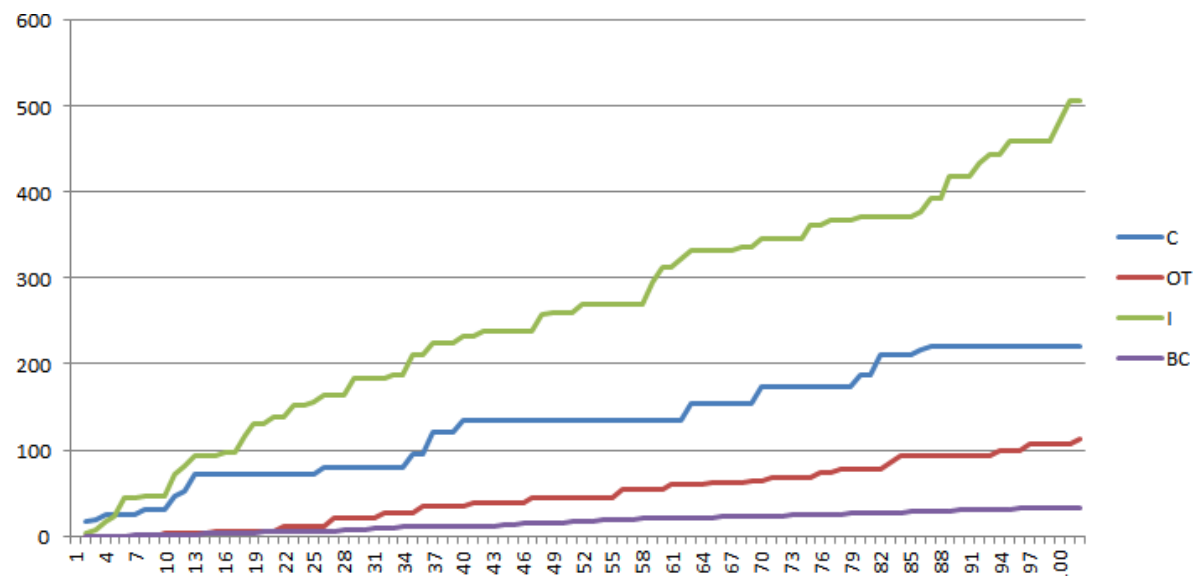

Figure 9. Events that affect the status of the robot during the second test of the second scenario

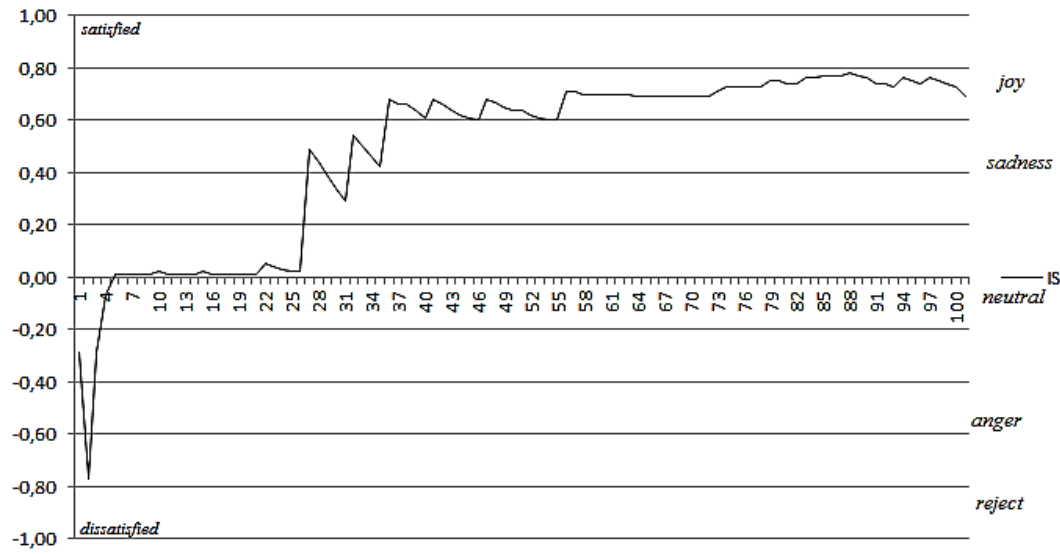

Figure 10. Results for the second test for the second scenario.

In Figure 10, it is observed that at time zero events occur that lead the robot to a highly negative emotional state, and then, a process begins that increases its state of satisfaction to stabilize in a state highly positive. This is explained by the events that occur that affect the state of the robot (see Figure 9), which corroborates that our emotional model considers the events in the context to determine the emotional state of the robot.

The previous tests show the emotional process that occurs in a robot during a period of time, and how the events that occur in the environment define its emotional state. According to the current emotional state, the behavior of the robot can be different (see section 3). If the robot has a positive emotional state, then it will have an imitative behavior, as, for example, to explore; if the robot has a highly negative emotion, then it will have a reactive behavior, for example, to avoid obstacles, and so for the rest of the emotional states. In this way is generated an emerging behavior in a robot, based on its current emotional state, which can change during its operation.

\subsection{Comparison with previous works}

In this section, a qualitative comparison of the proposed model with previous works is carried out, for which the following is taken into consideration (see Table 3): technique used, features that define the emotion, and the application of the emotional model. 
In [17], a system is implemented to identify the emotional state of a human being. For this, the signal coming from an electrocardiogram (ECG) is analyzed and a series of characteristics are extracted, which are processed in a hybrid system based on a back propagation neural network and a crossbar array. The set of identified emotions by the system are: \{angry, fear, grief, happy, and calm\}. Our work uses a fuzzy system with the goal of identifying the emotional state of a robot according to 4 factors that define its internal state. The identified emotion is not shown to the environment, but it is used individually to regulate the performance of the robot in the system. In both cases, the signal of the ECG and our 4 factors considered, are affected by the interaction of the individual/robot with the environment.

In [18], a neural network is used to identify emotions based on a two-dimensional model that encompasses a broad emotional spectrum, supported by the levels of pleasant-miserable, and arousal-sleepy of the individual, the system is designed for humans. In our model, a simplified one-dimensional spectrum is used, based on the level of satisfaction and dissatisfaction of the individual/robot.

The work presented in [19] implements in a mobile robot an emotional model that involves three aspects: emotion (taken as a stimulus), feeling (considered as a short-term state) and mood (long-term state). They regulate, as in our work, the behavior of the robot, affecting its ability to perceive and act. The robot interacts with the environment, the information received is processed by a neural network, and the result is the emotion that the robot will express during its interaction with the human beings. In our case, we use a simplified emotional spectrum \{happy, sad, fearful, angry\}, due to the robot's processing capabilities.

In [20], a fuzzy system is used for the recognition of human emotions from signals generated by an electroencephalogram (EEG), in order to be applied it in human-robot interaction systems. The characteristics used as inputs to the system are extracted of the analysis of the signal. In [21] is described a system for the recognition of emotions, also for the processes that occur in the human-robot interaction. The system allows identifying the next set of emotions \{sadness, angry, surprise, disgust, fear, neutral, happiness $\}$ in a human, from his facial gestures. In this model is present, as in our approach, the neutral state.

According to the Table 3, only our approach uses the emotions to regulate the behavior of the robots. The other approaches use the emotions for the human-computer interactions, they propose emotion recognition mechanisms for the human beings in order to guide the interaction of the robots with them. Our approach proposes an emotional model for the robots that determines their interactions and their influences on the environment, which are fundamental elements to generate an emergent behavior in a system $[12,16]$. Our model is very robust because defines the emotions of a robot based on its internal states, which characterize its real capabilities to interact in a given moment.

Table 3. Comparison with previous works

\begin{tabular}{cccc}
\hline Work & Technique used & Features to define an emotion & Application of the emotional model \\
\hline$[17]$ & $\begin{array}{c}\text { Back propagation neural network } \\
\text { and a crossbar array }\end{array}$ & Signals coming from an ECG & Human-robot interaction \\
{$[18]$} & Back propagation neural network & Colors as human behavior clues & Human-robot interaction \\
{$[19]$} & Spiking neural network & Gestural and facial expressions & Human-robot interaction \\
{$[20]$} & A fuzzy system & Signals coming from an ECG & Human-robot interaction \\
{$[21]$} & A fuzzy system & Facial expressions & Human-robot interaction \\
Our work & A fuzzy system & Internal state of the robot & To regulate the behavior of the robot \\
\hline
\end{tabular}

\section{CONCLUSION}

We have proposed an emotional model for an architecture of multi-robot systems with emergent behavior. The model simplifies the emotional spectrum based on four basic emotions: joy, sadness, rejection and anger. The model is implemented using a fuzzy model and is used for the generation of emergent behavior. Previous works have focused on the recognition of emotions, especially applied to human-robot interaction. In this work, the approach is oriented towards the ability to identify emotion as an individual state, which in principle affects the behavior of the robot. This influences the global behavior of the system, but not because individuals are able to recognize the emotion in the other, but because of the individual behavior that is generated in itself, which influences the global state. There is a robot-robot interaction explicitly directed by the current emotion in each robot.

The inclusion of emotions in the robot allows improving its adaptability to the dynamics of the environment, and facilitates the emergence and self-organization in a multi-robot system. Emotions influence the way that the robot acts according to the stimuli received, and can regulate its behavior 
over time. For example, if negative stimuli prevail during its performance, then the robot will take a certain emotional state for a certain time that will cause that it adapts itself to the environment. In this way, it will act according to its emotional state. For example, if the robot begins to collide constantly, then the robot will become cautious because its process of decision making will be cognitive due to its emotional state is sadness.

The expansion of the emotional spectrum and its implementation in robots with greater capacities, including the ability to express their emotional state, to allow other robots to recognize their state, are proposed as future works. Also, the analysis of the effects of the emotional state of the robots in the emergent behaviors in the system, must be carried out. The emotional states of the robots influence their interactions and with the environment, which should generate emergent behaviors in the system.

\section{REFERENCES}

[1] S. C. Banik, et al., "Behavior generation through interaction in an emotionally intelligent robot system," 2007 International Conference on Industrial and Information Systems, Penadeniya, 2007, pp. 517-522, doi: 10.1109/ICIINFS.2007.4579232.

[2] H. Hu, C. Gu, and H. Lian, "Design and Realization of Hardware System for Emotion Robot," in 2010 International Conference on E-Product E-Service and E-Entertainment, Henan, 2010, pp. 1-3, doi: 10.1109/ICEEE.2010.5661127.

[3] Q. Xiao and G. Ding, "A new emotional model based on multiple factors," in 2013 IEEE International Conference on Granular Computing (GrC), Beijing, 2013, pp. 357-361, doi: 10.1109/GrC.2013.6740436.

[4] C. M. Hsu, T. T. Chen, and J. S. Heh, "Emotional and Conditional Model for Pet Robot based on Neural Network," 2014 7th International Conference on Ubi-Media Computing and Workshops, Ulaanbaatar, 2014, pp. 305-308, doi: 10.1109/U-MEDIA.2014.33.

[5] C. P. Lee-Johnson and D. A. Carnegie, "Mobile Robot Navigation Modulated by Artificial Emotions," IEEE Transactions on Systems, Man, and Cybernetics, Part B (Cybernetics), vol. 40, no. 2, pp. 469-480, April 2010, doi: 10.1109/TSMCB.2009.2026826.

[6] A. Gil, et al., "Architecture for Multi-robot Systems with Emergent Behavior," in Proc. Int'l Conf. Artificial Intelligence, 2015, pp. 41-47.

[7] K. Hernández, et al., "Diseño de una plataforma Multi-Robot de Propósito General," in Proc. XIII Simulación y Aplicaciones Recientes para Ciencia y Tecnología, Sociedad Venezolana de Métodos Numéricos, vol. 8, pp. 785-796, 2016.

[8] A. Gil, et al., "A control architecture for robot swarms (AMEB)," Journal Cybernetics and Systems, vol. 50, no. 3, 300-322, 2019.

[9] A. Gil, et al., "Verification of the emergence in an architecture for multi-robot systems (AMEB)," Latin American Journal of Computing, vol. V, no. 2, pp. 41-52, 2018.

[10] A. Gil, et al., "Módulo conductual inmerso en una arquitectura de control para sistemas multi-robots," Ingeniería al Día, vol. 2, no. 1, pp. 40-57, 2016.

[11] N. Perozo, J. Aguilar, and O. Terán, "Proposal for a Multiagent Architecture for Self-Organizing Systems (MA-SOS)," Intelligence and Security Informatics, Lecture Notes in Computer Science, vol. 5075, pp. 434-439, 2008.

[12] N. Perozo, et al., "A Verification Method for MASOES," IEEE Transactions on Cybernetics, vol. 43, no. 1, pp. 64-76, Feb. 2013, doi: 10.1109/TSMCB.2012.2199106.

[13] N. Perozo, et al., "Un Modelo Afectivo para una Arquitectura Multiagentes para Sistemas Emergentes y Auto-organizados (MASOES)," Revista Técnica Facultad de Ingeniería, Universidad del Zulia, vol. 35, no. 1, pp. $80-90,2012$.

[14] R. Levenson, "The Intrapersonal Functions of Emotion," Cognition and emotion, vol. 13, no. 5, pp. 481-504, 1999.

[15] N. Perozo, et al., "Self-organization and Emergence Phenomena in Wikipedia and Free Software Development using MASOES," Publicaciones en Ciencias y Tecnología, vol. 7, no. 1, pp. 51-72, 2013.

[16] J. Aguilar, "Introducción a los Sistemas Emergentes," Talleres Gráficos, Universidad de Los Andes, 2014

[17] G. Xie, G. Liu and S. Zhang, "Expression of emotion using a system combined artificial neural network and memristor-based crossbar array," 2016 35th Chinese Control Conference (CCC), Chengdu, 2016, pp. 9837-9841, doi: 10.1109/ChiCC.2016.7554917.

[18] M. Lee and G. Chen, "Backpropagation neural network model for detecting artificial emotions with color," in 2013 International Joint Conference on Awareness Science and Technology \& Ubi-Media Computing (iCAST 2013 \& UMEDIA 2013), Aizu-Wakamatsu, 2013, pp. 433-438, doi: 10.1109/ICAwST.2013.6765479.

[19] J. Botzheim and N. Kubota, "Spiking neural network based emotional model for robot partner," in 2014 IEEE Symposium on Robotic Intelligence in Informationally Structured Space (RiiSS), Orlando, FL, 2014, pp. 1-6, doi: 10.1109/RIISS.2014.7009165.

[20] A. Chatchinarat, K. W. Wong, and C. C. Fung, "Fuzzy classification of human emotions using Fuzzy C-Mean (FCFCM)," in 2016 International Conference on Fuzzy Theory and Its Applications (iFuzzy), Taichung, 2016, pp. 1-5, doi: 10.1109/iFUZZY.2016.8004935.

[21] L. Chen, et al., "Dynamic Emotion Understanding in Human-Robot Interaction Based on Two-Layer Fuzzy SVR-TS Model," IEEE Transactions on Systems, Man, and Cybernetics: Systems, vol. 50, no. 2, pp. 490-501, 2020, doi: 10.1109/TSMC.2017.2756447. 


\section{BIOGRAPHIES OF AUTHORS}

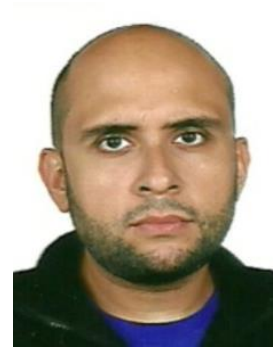

Angel Gil is a Computer Engineer from National Experimental University of Táchira, San Cristóbal, Venezuela. Obtainedan Advanced Studies Diploma in Systems and Automation Engineering in the University of Malaga, Spain. Doctor in the Science program Applied from the Universidad de los Andes, Mérida, Venezuela. He is a researcher / teacher in the Aggregate category at the University Nacional Experimental del Táchira (UNET), researcher of the Prototype Laboratory of the same university, from which it was responsible between 2010-2015. Coordinator of Industrial Research of the Research Dean UNET, 2015-2018 period. He has published around 30 articles in magazines and conference proceedings, in the area of AI, robotics and software development. He is currently a developer of software at the company AlquimiaSoft. S.A. in Ambato, Ecuador and member of the Tepuy R + D Research Network Group, Artificial Intelligence Software Development.

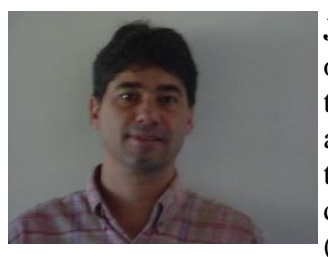

Jose Aguilar is a System Engineer from the University of the Andes, Mérida, Venezuela. He obtained a Master in Computer science at the Paul Sabatier University, Toulouse, France and the Doctorate in Sciences Computacionales at Rene University Descartes, Paris, France. In addition, he completed a Postdoctoral degree in the Department of Computer Sciences of the University of Houston. He is Professor of the Department of Computing of the Universidad de los Andes (ULA) and researcher at the Center for Microcomputing and Systems Distributed (CEMISID) from the same university. Is a member Corresponding State of the Academy of Merida and the IEEE International Technical Committee on Neural Networks. In addition, he is former coordinator of the Doctoral Program in Applied Sciences from the Faculty of Engineering of the University of the Andes. He was founding President of the Center National Development and Research in Free Technology (CENDITEL), and from FUNDACITE-Mérida. In addition, Head of ULA Department of Computing from 2011 to 2014, and Director of CEMISID from 2001 to 2007 . He has published more than 400 scientific articles in magazines, books and conference proceedings international, in the fields of Parallel Systems and Distributed, Smart Computing, Optimization Combinatorics, Pattern Recognition, Systems Industrial Automation, etc. He has also been an author/co-author of 10 books, editor of several books, and is part of severa magazine editorial committees. In addition, he has taught courses in national and international training and has received several national and international awards/recognitions scientists. Dr. He has been president of the Scientific Committee of various national and international events. Furthermore, it has coordinated or participated in more than 30 projects research or industrial. On the other hand, he has been a consultant for different companies, ministries and universities Latin American and Venezuelan. Finally, he has been tutor of more than 90 Bachelor, Master and Doctorate theses, he is currently coordinator of the Tepuy Research Network R + D Group, Artificial Intelligence Software Development.

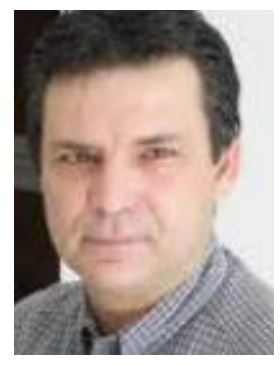

Eladio Dapena is a Systems Engineer from the University of Los Andes (ULA), Mérida, Venezuela. Doctorate in Industrial Engineering and DEA from the Carlos III University of Madrid, Spain, Has Specialization in Industrial Automation by the University Federal de Santa Catarina (UFSC), Brazil. He is a titular professor of the ULA and visiting professor / researcher in several universities in the world with 25 years of experience. Directs for 15 years the LaSDAI Research Laboratory of the ULA Faculty of Engineering developing projects $\mathrm{R}+\mathrm{D}+\mathrm{i}$ in the areas of industrial automation and robotics. He has various publications and is a reviewer for different magazines and committees in the areas of Robotics and Automation. Tutor / co-tutor of more than 20 Bachelor's, Master's and Doctorate. He has participated in various curricular reviews and creator of the first Diploma in Robotics in Venezuela. He has participated in Projects / Consulting in various industries. He has also held university management positions. It was Founding President of the Venezuelan Robotics Association and Domotics (AVEROD).

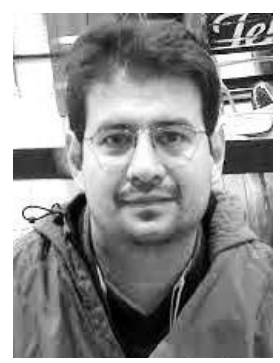

Rafael Rivas is a Systems Engineer and Magister Science from the University of Los Andes, Mérida, Venezuela. DEA in Engineering of Systems from the Carlos III University of Madrid Spain. He completed his PhD in Engineering at the Carlos III University of Madrid. His $\mathrm{PhD}$ thesis was in the area of Architectures control for Personal Mobile Robots. It's one of the creators of the Robot Maggie from UC3M. He is a full professor attached to the Computer Department of the University of Los Andes, Merida, Venezuela. 